\title{
The Chlorthalidone Saga: How the US Medical Community was mislead in Past?
}

\author{
Franz H Messerli
}

Keywords: Chlorthalidone, Hydrochlorothiazide, Thiazides.

How to cite this article: Messerli FH. The Chlorthalidone Saga: How the US Medical Community was mislead in Past? Hypertens J 2016;2(4):209-210.

\section{Source of support: Nil}

\section{Conflict of interest: None}

\section{INTRODUCTION}

In this issue of the journal Pareek and colleagues provide a thorough, comprehensive review article on chlorthalidone (CTD). They report that in various global large trials like TOMHS, MRFIT, SHEP, ALLHAT, and SPRINT, CTD has reduced cardiovascular $(\mathrm{CV})$ events, strokes, and, most importantly, mortality across various patient subgroups. Not surprisingly, documentation of such consistent benefits seems to finally have led to a renaissance of a drug that was launched more than a century ago. Since apart from SPRINT, all CTD studies have been around for years and decades, one may ask why exactly it took so long to recognize the unsurpassed benefits of CTD and to translate them into clinical practice. When attempting to analyze this question, it becomes clear that the major reason for the delay was the deceptive information policy of the National Heart Lung and Blood Institute (NHLBI).

Hydrochlorothiazide (HCTZ) remains by far the most commonly prescribed antihypertensive agent in the USA and worldwide. This is not surprising, since starting with Joint National Committee (JNC) I, every subsequent JNC advocated "thiazides" for the first-line therapy for hypertension by stating that thiazides should be preferred over other drugs because they had been shown to reduce morbidity and mortality. The NHLBI and the authors of various JNCs thereby tacitly implied that thiazides were synonymous with HCTZ. Before we continue to subscribe

Professor

Department of Cardiology, Icahn School of Medicine at Mount Sinai, New York; University of Bern, Switzerland and Jagiellonian University, Kraków, Poland

Corresponding Author: Franz H Messerli, Professor, Department of Cardiology, Icahn School of Medicine at Mount Sinai, New York; University of Bern, Switzerland and Jagiellonian University Kraków, Poland, e-mail: messerli.f@gmail.com to such profound wisdom, we should consider the following simple facts:

- Hydrochlorothiazide is one of the weakest antihypertensive agents available. In head-to-head comparison by 24-hour ambulatory monitoring, the antihypertensive efficacy was shown to be inferior to other drug classes, such as angiotensin-converting enzyme inhibitors, angiotensin receptor blockers, beta-blockers, and calcium channel blockers.

- Hydrochlorothiazide's antihypertensive effect does not last 24 hours, thereby leaving the critical early morning hours unprotected. With HCTZ therapy, sustained hypertension merely will be converted into masked hypertension. Blood pressure (BP) seems to be well controlled during daytime, when patients are seen in the office. However, as shown in Graph 1 (See page 204) of Pareek et al, late night-to-early morning BP remains poorly controlled by HCTZ. Importantly, this time period has been identified as the most critical in the diurnal cycle because it coincides with the highest risk of stroke and other CV events. As shown in the same figure, CTD provides smooth BP control throughout a full 24-hour period.

- Hydrochlorothiazide in its usual dose of 12.5 to $25 \mathrm{mg}$ per day has never been shown to reduce the risk of myocardial infarction, stroke, or death. In fact, higher doses have been shown to increase the risk of sudden cardiac death in a dose-dependent fashion.

- Even in combination with an angiotensin-converting enzyme inhibitor, HCTZ has been shown to be inferior to a calcium channel blocker, such as amlodipine. In the avoiding cardiovascular events through combination therapy in patients living with systolic hypertension (ACCOMPLISH) trial, despite identical BP reduction, the amlodipine-based combination reduced CV events by $20 \%$ better than the HCTZ combination, a fact that led to a premature termination of the study.

- All efficacy and outcome data for thiazides are solely derived from CTD and indapamide. However, both of these drugs, CTD and indapamide, are distinctly different from HCTZ and have been documented to exert pleiotropic effects and a longer duration of action that may account for their superior efficacy. The NHLBI has continued to deceptively promote "low-dose thiazides" based on the CTD data, although tacitly clearly aware that such promotion will only motivate physicians to treat more and more patients with HCTZ. 
There is no question that HCTZ should be avoided as the first-line therapy for hypertension. If a thiazide is deemed to be appropriate, our choice should be CTD or indapamide, as is stated now in several recent sets of guidelines. The calamity of millions of patients being exposed to an inefficacious drug could have been avoided if the NHLBI simply had stated the facts, namely, that CTD and not HCTZ or a "thiazide" was the drug that time and again reduced morbidity and mortality in all studies. Conceivably, several hundred thousands of strokes and heart attacks could have been avoided in the USA alone by a straightforward information policy of the NHLBI. If the data are achieved with CTD and not with HCTZ, the NHLBI had and still has an ethical obligation to say so. Given this background, the JNC 7 statement "diuretics have been virtually unsurpassed in preventing the cardiovascular complications of hypertension" must be considered to be purposefully deceptive. Clearly for many years the NHLBI has sold American physicians and patients down the river.

Pareek et al (See article "Renaissance of Chlorthalidone") concluded their comprehensive review on CTD with the simple statement: "The dose of Chlorthalidone $6.25 \mathrm{mg}$ is most suitable for Indian patients. Published Indian evidence indicates that this low dose of chlorthalidone could reduce dose-related concerns about metabolic adverse effects and may lead to its wider usage as an antihypertensive agent."

There is no valid reason for not accepting these facts for the USA as well and put them to widespread use in treating our patients. 